\title{
EXPERIENCE WITH THE BEAM POSITION MONITOR SYSTEM OF THE BESSY II STORAGE RING*
}

\author{
$\underline{\text { P. Kuske }}{ }^{\#}$, R. Bakker, F. Falkenstern, R. Görgen, D. Krämer, J. Kuszynski, R. Müller \\ BESSY, Berlin, Germany
}

\begin{abstract}
The beam position monitor (BPM) system has been developed at BESSY and operates in 3 different modes: the closed orbit, the single turn, and the diagnostics mode. Details of the BPM system are presented together with the result of some bench tests. The commissioning of the system was done primarily with beam-based techniques and is described in more detail. First measurements of fast and slow beam motions are presented. From the very beginning the BPM system was used successfully for the optimisation of the storage ring.
\end{abstract}

\section{INTRODUCTION}

Stability of the beam position in a third generation light source like BESSY II [1] is essential in order to achieve and guarantee the high brilliance of the photon beams produced by insertion devices. Usually a lot of care is taken in the construction of the facility and in the design of the hardware to prevent beam motion from environmental or other sources. In any case a fast and accurate measurement of the orbit is required for monitoring beam motion or use the measurements in order to improve the short and long term position stability with local or global feedback systems. Aside from these more user driven requirements, the measurement of orbits and their variation as other storage ring parameters are changed, i. e. steering magnets for the determination of the response matrix or individual quadrupole magnets for beam-based alignment, are very important for understanding the storage ring hardware, the optics and for improving the performance of the storage ring as a light source. Accelerator physicists usually expect in addition to the precise orbit measurements a turn-by-turn capability or in the case of BESSY a diagnostics mode of operation.

\section{TECHNICAL BPM DESIGN}

The general technical layout of the BPM system [2] chosen for the BESSY II storage ring is shown in Fig. 1. The basic operating principle for closed orbit measurements has been developed at the NSLS storage ring [3]. The four pickup signals are attenuated and

"Work supported by the Bundesministerium für Bildung, Wissenschaft, Forschung und Technologie and by the Land Berlin

\# Email: Kuske@bii.bessy.de

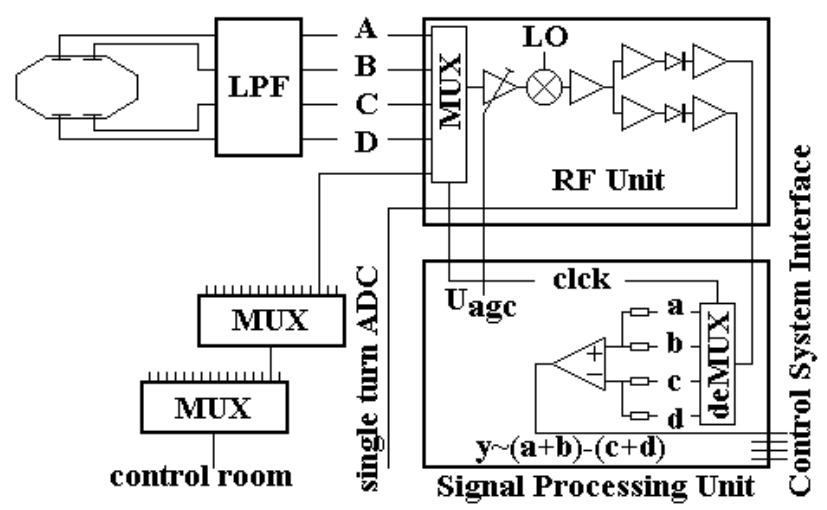

Figure 1: Layout of the BESSY II BPM system.

filtered in the low-pass filter (LPF) located close to the sensor and switched at a rate of $4 \mathrm{kHz}$ sequentially to the tuned receiver contained in the RF unit. The rectified output of the super heterodyne receiver is processed in the signal processing unit by purely analog means. This is indicated in Fig. 1 for the vertical position of the beam. Finally both positions, the gain control voltage, Uagc, which is used to keep the sum signal constant, and an error signal proportional to (A-C)-(B-D) are available in analog form. All signals are digitised at a rate of $10 \mathrm{kS} / \mathrm{s}$ with 16 bit resolution. Usually each input is sampled over $100 \mathrm{~ms}$ and then averaged. Mean values, standard deviations, and status bits are available in the control system. A new orbit can be displayed every second.

The receiver has a second demodulated output with much larger bandwidth of $1.5 \mathrm{MHz}$ which is used for turn-byturn measurements. 64 such signals are digitised over 32 thousand turns with 12 bit resolution. This is repeated for each pickup signal at $100 \mathrm{~ms}$ intervals in synchronism with the injection system running at $10 \mathrm{~Hz}$. The position of the beam is calculated by a computer. Displayed orbits on a pre-selected turn are updated every 2 seconds.

Alternatively, in the diagnostics mode, the signal of a particular pickup electrodes can be switched to the control room through a chain of 16-to-1 multiplexers (MUX).

\section{DEBUGGING AND COMMISSIONING OF THE BPM SYSTEM}

Prior to installation all units and pieces of equipment relevant for the precise and stable operation of the BPM systems were carefully checked on the bench whenever reasonable. In addition strong emphasis was put on the 
commissioning of the complete system with beam-based techniques.

\subsection{Bench tests}

For example the test of the short connection between the pickup electrodes and the LPF box with high quality semi rigid SMA-cables were impossible since their installation had to be done just before the vacuum chamber was lowered into the half-opened magnets and finally fixed to the girders. During that moment of time careful RF measurements with a network or spectrum analyser were impossible. Later on it turned out, that 5 out of the 112 BPMs could not be used, because of faulty connections of that type. During the last shut down period 3 of them could be fixed. In the two other cases the vacuum feed through of the pickup would have to be replaced, which is, at least in principle, possible since the pickups are welded into demountable flanges. For the time being the beam positions at these locations are determined from only 3 pickup signals by a small modification to the signal processing electronics.

The results of the bench tests can be summarised as follows: in the closed orbit mode the position of the beam can be measured over a very large range of intensities spanning more than 4 orders of magnitude. The measurements are insensitive to the beam intensity over a range of nearly 3 orders of magnitude at the few micron level even for beams being off-centre by $\pm 5 \mathrm{~mm}$. At nominal beam currents, above $50 \mathrm{~mA}$, the resolution is better than $1 \mu \mathrm{m}$ in a $1 \mathrm{~ms}$ time interval. Fast transverse beam oscillations up to $250 \mathrm{~Hz}$ can be observed. In the single turn mode the resolution at low intensity is $\approx 1 \mathrm{~mm}$. With the expected large excursions of the injected beam and without correction the non-linear contributions from the vacuum chamber geometry and the arrangement of the pickup electrodes is much larger than the resolution. In the diagnostics mode the filtered pickup signals in the control room are attenuated by approximately $20 \mathrm{~dB}$ due to the losses in the LPF, insertion losses of the chain of multiplexers, and cable losses.

\subsection{System tests without beam}

Unexpectedly difficulties arose with the local oscillator due to a poor quality inductor and subsequently the coil had to be replaced in nearly all oscillators. In this debugging phase all components of the BPM system were already installed and the diagnostics mode could be used for trouble shooting: In the control room a $500 \mathrm{MHz}$ signal at $0 \mathrm{dBm}$ from the master oscillator was fed into the output of the chain of RF multiplexers. The BPM system was set to run in the closed orbit mode and with the finite isolation of the last GaAs-switch the selected unit should work properly showing a nicely centred beam position. The procedure was automated and for each BPM the signals to the control system were recorded. Thus at the same time the proper operation of the BPM system including the control system interface was checked.

\subsection{Commissioning with beam}

During the first 24 hours of the storage ring commissioning an electron beam could be stored without using the turn-by-turn mode only with the support of 9 fluorescent screens distributed around the circumference of the ring. Due to the large dynamic range of the BPM electronics, orbits could be measured at currents above 50 $\mu \mathrm{A}$ which is achievable with single shot injection. Thus a lot of the final commissioning of the BPM system running in the closed orbit mode was done with stored beam. In the beginning with closed orbit distortions of up to $\pm 10 \mathrm{~mm}$ the error signal was very helpful in order to find missing connections and interchanged cables. In this way a couple of inoperable BPMs could be excluded for example from the closed orbit correction algorithm or the analysis of collected orbit data.

Another very helpful tool is the analysis of measured response matrices [4]. At BESSY II this matrix contains 112 times 145 elements. Each element is the orbit variation measured at one of the 112 BPMs as the 145 steering elements are changed by a small amount. The lattice and the arrangement of hardware is of 8 -fold symmetry. Thus every eighth column should contain very similar information. These 8 columns can be displayed superimposed if the numbering is shifted correspondingly. Individual hardware errors show up as large deviations from the average. In this way the gain error of one of the signal processing boards could be detected.

Total BPM offset errors with respect to the centres of nearby quadrupole magnets have been obtained with an iterative procedure developed and tested at BESSY I [5]. Additional windings on the 144 quadrupole magnets of the BESSY II storage ring allow to change the gradient in each magnet. The resulting closed orbit perturbations are proportional to the actual offsets of the orbit relative to the axis of the magnet. This information has been used to redefine the targets for the closed orbit correction. In the horizontal plane a few iterations were required in order to achieve a centred beam in all quadrupoles. Finally the beam is on average only off by $\sigma y=0.13 \mathrm{~mm}$ and $\sigma x=0.15$ $\mathrm{mm}$ relative to these magnets. The resulting distributions of the total BPM offset errors have mean values of -0.02 $\mathrm{mm}$ and $0.28 \mathrm{~mm}$ and standard deviations of $0.24 \mathrm{~mm}$ and $0.43 \mathrm{~mm}$ in the horizontal respectively in the vertical plane. This spread is in agreement with expectations based on the bench measurements and reasonable assumptions for the precision of the mechanical adjustments of the pickup sensors and the limitations of the steering concept based on 64 vertical and 80 horizontal corrector magnets. The significant mean offset in the vertical plane indicates that the vacuum chambers have been mounted too low. 

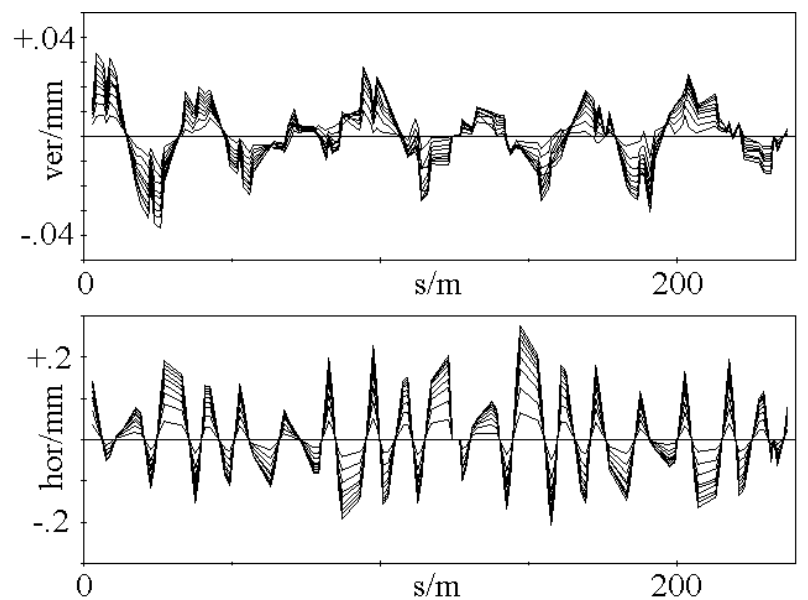

Figure 2: Slow orbit drifts in the vertical (top) and horizontal plane (bottom) relative to the orbit just after a beam of $200 \mathrm{~mA}$ was accumulated.

\section{OPERATIONAL EXPERIENCE}

As expected the most important mode of operation is the measurement of the closed orbit. Single turn data has only been used a few times early during the commissioning of the storage ring and was helpful to give the integer part of the tunes [2]. Until now the diagnostics mode has only been applied for trouble shooting the BPM system.

Software tools have been developed in order to collect orbit data at a rate of 1 orbit per second while other parameters are varied. Examples are the above mentioned beam-based determination of the position relative to the quadrupole magnets or the measurement of the response matrix. Both measurements take 1 to 2 hours depending on the degree of averaging chosen. These and similar tools have been extremely helpful for the smooth and fast commissioning of the BESSY II storage ring [6].

Slow and intensity dependent orbit drifts can be observed in both planes during the daily operation of the ring. In the case shown in Fig. 2 the beam was accumulated within less than 2 minutes from $10 \mathrm{~mA}$ to nearly $200 \mathrm{~mA}$. The orbits have been taken just after the injection in 1 minute intervals. During the injection the beam motion was small. Drifts are much larger horizontally and an equilibrium is reached after approximately 10 minutes. This and the repetition of the pattern of the orbit distortion after each refill is an indication that they are induced by thermal effects. In routine operation the drifts can be compensated for by running the global orbit correction permanently.

Fast oscillations of the closed orbit can not be observed directly since the presently installed control system interface is too slow. Additional buffered outputs are available for the off-line analysis at each signal processing unit. In Fig. 3 the FFT of the horizontal beam motion at a location with high dispersion is shown. The observations were made at high and low beam currents, before and after the modification of the horizontal steering magnet power supplies and the installation of

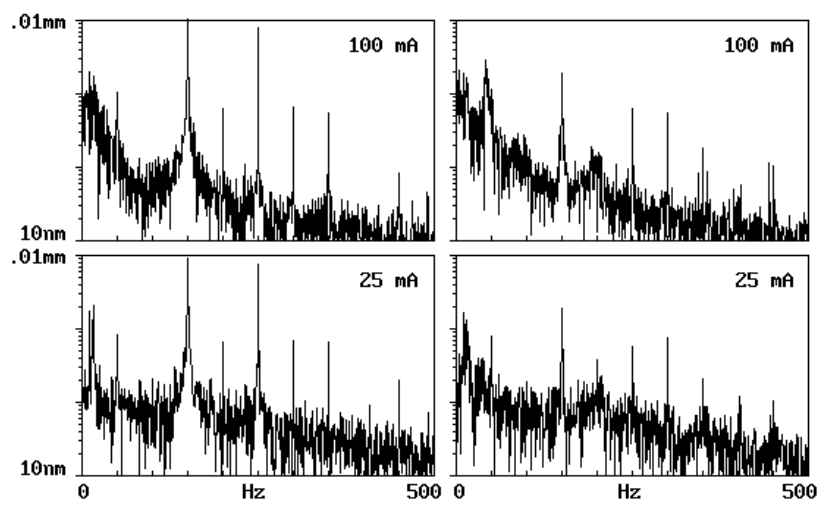

Figure 3: Fast beam motion in the horizontal plane before (left) and after hardware modifications. The top traces are taken at around $100 \mathrm{~mA}$ and the lower traces at $25 \mathrm{~mA}$.

damping antennas in all 4 cavities. The impact of the synchrotron running at $10 \mathrm{~Hz}$ and eigen modes of the storage ring girders at around $15 \mathrm{~Hz}$ are visible at low intensities. At high beam currents longitudinal instabilities produce a broad low-frequency noise floor which does not appear in the vertical plane.

\section{SUMMARY}

The BPM system of the BESSY II storage ring has been in operation from the very beginning of the commissioning. In addition to bench tests further debugging took place with beam-based techniques. In the closed orbit mode beam positions are measured fast, accurate, insensitive to changing beam currents, and over a very large dynamic range of intensities. 64 out of the 112 BPMs have a limited turn-by turn capability and the diagnostics mode can be used for the analysis of the 500 $\mathrm{MHz}$ component of the beam spectrum by special equipment in the control room. The system is in full operation now and has led to substantial progress already.

\section{ACKNOWLEDGEMENT}

The support of the BESSY staff in the operation of the facility and in the acquisition of the data is gratefully acknowledged.

\section{REFERENCES}

[1]W. Anders, et. al. "Status And Commissioning-Results Of BESSY II", this conference

[2]R.J. Bakker, et. al. "Fast And Flexible BPM-System: Valuable Commissioning Tool for BESSY II", EPAC 98, Stockholm, p. 1482, (1998)

[3]R. Biscardi, J.W. Bittner, "Switched Detector For Beam Position Monitor", PAC'89, Chicago, Vol. 3, p. 1516, (1989)

[4]W.J. Corbett, et. al., "Debugging Real Accelerators", Particle Accelerator, 1997, Vol. 58, p. 193

[5]P. Kuske, K. Ott, "Beam-Based Alignment At BESSY, EPAC'96, Sitges (Barcelona), Vol. 2, p. 887, (1986)

[6]P. Bakker, et. al., "Experiences With Commissioning Software Tools At BESSY II, this conference 